\title{
On the Molecular Packing Density in Crystalline Polymers
}

\author{
V. P. Privalko \\ Institute of Macromolecular Chemistry, Academy of \\ Sciences of the Ukrainian SSR, 252160, Kiev 160, \\ Kharkovskoye Chaussee, 48, USSR.
}

(Received June 17, 1974)

\begin{abstract}
Values of coefficients of crystalline packing density, $K_{\mathrm{c}}$, calculated for a large number of polymers with chain backbones consisting of single carbon-carbon, carbon-oxygen, etc., bonds, exhibit strong dependence on polymer chain conformation in the crystalline state. It was established that $K_{\mathrm{c}}$ values tend to decrease regularly with the increase of chain "thickness" expressed by molecular cross-section, $A$, for polymers with helical conformation, while the reverse was true for extended-chain polymers. This difference is consistent with previously obtained results and can be derived analytically.
\end{abstract}

\section{KEY WORDS Crystalline Polymers / Molecular Packing Density /}

Chain Conformation /

As shown by Kitaigorodskii, ${ }^{1,2}$ the necessary prerequisite for the existence of a thermodynamically stable crystal is the minimal energy of intermolecular interaction, which is achieved in the state of the most dense packing of molecules in the crystalline lattice. The efficiency of molecular packing in the crystal is expressed through the so-called packing coefficient, $K_{\mathrm{c}}$, which is defined by eq $1::^{1,2}$

$$
K_{\mathrm{c}}=V_{0} N_{\mathrm{A}} / V_{\mathrm{c}}
$$

In this equation $V_{0}$ is the "intrinsic" molecular volume corresponding to the one obtained by framing the molecule with its intermolecular (Van der Waals) radii, $V_{\mathrm{c}}$ is the molar volume of a substance in the crystalline state, and $N_{\mathrm{A}}$ is Avogadro's number. For the majority of molecular crystals the numerical values of $K_{\mathrm{c}}$ vary within the relatively narrow interval from 0.68 to $0.75 ;^{1,2}$ these are close to the value 0.74 characteristic to the dense packing of spheres of ellipsoids of rotation. For polymers, on the other hand, the dependence of $K_{\mathrm{c}}$ on the chemical nature of the chain has been studied much less. Starting from the premise that the packing coefficient of amorphous polymers at room temperature is approximately invariant, ${ }^{3,4}$ Bessonov, et $a l .{ }^{5}$ and Van Krevelen ${ }^{4}$ suggested that for polymeric substances the value of $K_{\mathrm{c}}$ is also a "universal" constant equal either to $0.71^{4}$ or to
0.74. ${ }^{5}$ However, as follows from the more detailed analysis, ${ }^{6-9}$ the numerical values of $K_{\mathrm{c}}$ for polymers vary over a rather wide interval, while a definite trend to lower $K_{\mathrm{c}}$ values with an increase of the dimensions of the side groups was observed..$^{6,9}$ It is the purpose of this paper to explore the existing situation more carefully and to show that the variation of $K_{\mathrm{c}}$ values among different polymers is related to the intrinsic differences in chain conformations in the crystals.

\section{DISCUSSION}

Values of $K_{\mathrm{c}}$ for a large number of polymers with chain backbone composed of single carboncarbon, carbon-oxygen, or carbon-nitrogen bonds are listed in the Table I. For reasons which will become evident from subsequent discussion and have been more fully explained elsewhere ${ }^{10,11}$ polymers with double bonds and/or phenyl rings in the main chain were not considered here. To calculate $K_{\mathrm{c}}$ values for polymers listed in Table I, tabulated values of $V_{0}^{2,3}$ and $V_{\mathrm{c}}{ }^{12}$ were used throughout.

It is easily seen from the table that the variations of $K_{\mathrm{c}}$ values for polymers are much broader (from 0.6 to 0.83 ) than those for low-molecularweight compounds. Therefore, any postulated invariance of $K_{\mathrm{c}}$ for polymers ${ }^{4,5}$ is clearly 
On the Molecular Packing Density in Crystalline Polymers

Table I. Values of $V_{0}^{2,3}$, crystalline density $\rho_{\mathrm{c}}, A^{12}$, and $K_{\mathrm{c}}$

\begin{tabular}{|c|c|c|c|c|c|c|c|}
\hline No. & Chain repeating unit & $\begin{array}{l}\rho_{\mathrm{c}}, \\
\mathrm{g} / \mathrm{cc}\end{array}$ & $\begin{array}{c}V_{0}, \\
\mathrm{cc} / \mathrm{mol}\end{array}$ & $\begin{array}{l}A, \\
\AA^{2}\end{array}$ & $K_{\mathrm{c}}$ & $\underset{\text { type }}{\text { Helix }}$ & $\begin{array}{c}\text { Type of } \\
\text { chain }\end{array}$ \\
\hline \multicolumn{8}{|c|}{ Polyolefins } \\
\hline 1 & Butene-1 (form 1) & 0.95 & 41.4 & 45.5 & 0.701 & $3 / 1$ & $\mathbf{b}$ \\
\hline 2 & Butene-1 (form 2) & 0.886 & 41.4 & 60.6 & 0.655 & $4 / 1$ & $\mathrm{c}$ \\
\hline 3 & 3-Methylbutene-1 & 0.92 & 51.9 & 74.2 & 0.683 & $4 / 1$ & c \\
\hline 4 & Ethylene & 1.0 & 20.6 & 18.2 & 0.735 & $1 / 1$ & a \\
\hline 5 & Chlorotrifluoroethylene & 2.19 & 39.9 & 31.6 & 0.750 & $14 / 1$ & a \\
\hline 6 & Tetrafluoroethylene & 2.4 & 34.4 & 28.2 & 0.825 & $13 / 6$ & a \\
\hline 7 & Hexadecene-1 & 0.95 & 159.5 & 234.5 & 0.675 & $4 / 1$ & c \\
\hline 8 & Hexene-1 & 0.91 & 61.8 & 78.0 & 0.670 & $7 / 2$ & $\mathbf{b}$ \\
\hline 9 & 4-Methylhexene-1 & 0.845 & 72.8 & 96.5 & 0.626 & $7 / 2$ & b \\
\hline 10 & 5-Methylhexene-1 & 0.84 & 72.8 & 96.9 & 0.623 & $3 / 1$ & $\mathbf{b}$ \\
\hline 11 & Isobutylene & 0.915 & 41.6 & 43.4 & 0.695 & $8 / 3$ & $\mathbf{b}$ \\
\hline 12 & Octadecene-1 & 0.95 & 185.1 & 267.2 & 0.697 & $4 / 1$ & c \\
\hline 13 & Pentene-1 (form 1) & 0.96 & 51.6 & 56.1 & 0.708 & $3 / 1$ & $\mathrm{~b}$ \\
\hline 14 & Pentene-1 (form 2) & 0.885 & 51.6 & 74.8 & 0.663 & $4 / 1$ & c \\
\hline 15 & 4-Methylpentene-1 & 0.816 & 62.2 & 86.5 & 0.604 & $7 / 2$ & $\mathrm{~b}$ \\
\hline 16 & Propylene & 0.94 & 31.1 & 34.3 & 0.700 & $3 / 1$ & $\mathrm{~b}$ \\
\hline 17 & Tetradecene-1 & 0.95 & 143.9 & 208.0 & 0.696 & $4 / 1$ & c \\
\hline 18 & Vinylcyclohexane & 0.95 & 74.5 & 119.5 & 0.643 & $4 / 1$ & c \\
\hline \multicolumn{8}{|c|}{ Polyvinyls and Polyvinylidenes } \\
\hline 19 & sec-Butyl acrylate & 1.06 & 77.1 & 93.1 & 0.636 & $3 / 1$ & b \\
\hline 20 & Isopropyl acrylate & 1.08 & 66.9 & 80.2 & 0.635 & $3 / 1$ & $\mathrm{~b}$ \\
\hline 21 & tert-Butyl acrylate & 1.04 & 77.1 & 95.3 & 0.637 & $3 / 1$ & b \\
\hline 22 & Acrylonitrile & 1.27 & 32.6 & 29.1 & 0.766 & $1 / 1$ & $\mathrm{a}$ \\
\hline 23 & Methyl methacrylate & 1.23 & 58.5 & 63.3 & 0.715 & $5 / 1$ & $\mathbf{b}$ \\
\hline 24 & Vinyl alcohol & 1.35 & 25.1 & 21.6 & 0.766 & $1 / 1$ & $\mathrm{a}$ \\
\hline 25 & Vinyl chloride & 1.53 & 29.5 & 28.6 & 0.724 & $1 / 1$ & a \\
\hline 26 & Vinyl fluoride & 1.44 & 22.5 & 21.7 & 0.705 & - & $\mathbf{b}^{\mathrm{a}}$ \\
\hline 27 & Vinylidene bromide & 3.065 & 44.5 & 42.3 & 0.735 & - & $b^{a}$ \\
\hline 28 & Vinylidene chloride (form 1) & 1.96 & 38.6 & 35.2 & 0.778 & $1 / 1$ & $\mathrm{a}$ \\
\hline 29 & Methyl vinyl ketone & 1.216 & 42.1 & 46.4 & 0.733 & $7 / 2$ & b \\
\hline \multicolumn{8}{|c|}{ Polyaromatics } \\
\hline 30 & Styrene & 1.12 & 66.0 & 70.5 & 0.705 & $3 / 1$ & $\mathbf{b}$ \\
\hline 31 & $o$-Fluorostyrene & 1.296 & 71.5 & 71.5 & 0.760 & $3 / 1$ & b \\
\hline 32 & $o$-Methylstyrene & 1.07 & 80.2 & 100.2 & 0.660 & $4 / 1$ & c \\
\hline 33 & $m$-Methylstyrene & 1.02 & 80.2 & 107.9 & 0.630 & $11 / 3$ & c \\
\hline 34 & 1-Vinylnaphthalene & 1.12 & 85.6 & 114.2 & 0.623 & $4 / 1$ & c \\
\hline \multicolumn{8}{|c|}{ Polyamides } \\
\hline 35 & $\varepsilon$-Caprolactam & 1.23 & 69.6 & 20.3 & 0.758 & $1 / 1$ & a \\
\hline 36 & 8-Aminocaprylic acid & 1.18 & 90.2 & 20.3 & 0.755 & - & $\mathrm{a}^{\mathrm{a}}$ \\
\hline 37 & 7-Aminoenantic acid & 1.2 & 79.9 & 20.3 & 0.756 & - & $a^{a}$ \\
\hline 38 & 11-Aminoundecanic acid & 1.168 & 121.1 & 20.5 & 0.775 & - & $a^{a}$ \\
\hline 39 & Hexamethylene adipamide & 1.24 & 139.2 & 19.9 & 0.775 & - & $a^{a}$ \\
\hline 40 & Hexamethylene sebacamide & 1.17 & 177.7 & 20.3 & 0.735 & - & $\mathrm{a}^{\mathrm{a}}$ \\
\hline \multicolumn{8}{|c|}{ Polyesters and Polyethers } \\
\hline 41 & Ethylene adipate & 1.34 & 98.5 & 19.9 & 0.770 & $1 / 1$ & a \\
\hline 42 & Ethylene azelaate & 1.22 & 128.8 & 18.6 & 0.735 & $1 / 1$ & a \\
\hline 43 & Ethylene sebacate & 1.148 & 139.1 & 18.8 & 0.732 & $1 / 1$ & $\mathrm{a}$ \\
\hline 44 & Ethylene suberate & 1.281 & 118.7 & 20.3 & 0.760 & - & $a^{a}$ \\
\hline 45 & Ethylene succinate & 1.358 & 78.3 & 20.1 & 0.739 & - & $\mathrm{a}^{\mathrm{a}}$ \\
\hline 46 & Ethylene oxide & 1.33 & 25.5 & 21.5 & 0.745 & $7 / 2$ & b \\
\hline 47 & Methylene oxide & 1.53 & 16.1 & 17.2 & 0.802 & $9 / 5$ & b \\
\hline 48 & Propylene oxide & 1.102 & 35.2 & 24.5 & 0.670 & $2 / 1$ & b \\
\hline 49 & Tetramethylene oxide & 1.12 & 46.9 & 17.3 & 0.730 & $1 / 1$ & a \\
\hline
\end{tabular}

a Tentative assignment.

b Glide form. 


\section{P. Privalko}

erroneous. It is also worth noting that the maximum value of $K_{\mathrm{c}}(0.83)$ for polymers is much higher than that for low-molecular-weight crystals (0.77). In our opinion, this latter effect may be explained as follows. In the case of low-molecular-weight substances the distance between the neighboring molecules in any direction of the lattice cannot be less than the sum of their intermolecular radii (in other words, molecules can not "interpenetrate"). On the other hand, in the case of polymers, the distances between the centers of atoms along the backbone are substantially less than the sum of corresponding Van der Waals-radii, thus leading to a more compact structure with higher $K_{\mathrm{c}}$.

Prior to the analysis of the $K_{\mathrm{c}}$ values listed in the table, it should be recalled that the chainlike structure of all the polymers requires that the packing of macromolecules into the crystal lattice must be with their long axes parallel to each other. Therefore, one must distinguish between the chain packings in at least two directions; these are longitudinal (intramolecular packing) and transverse (intermolecular packing). We may assume for simplicity that the transverse packing of macromolecules of different polymers in the lattice will resemble the spatial arrangement of intermeshing screws, ${ }^{13}$ where the bulges of each molecule fit the appropriate cavities in the neighboring one. Evidently, conditions for the transverse packing of chains cannot drastically differ, at least among polymers with similar cohesive energies of the crystal. Thus, the observed variation of $K_{\mathrm{c}}$ for polymers should be attributed primarily to the intrinsic differences in intramolecular packing (i.e. chain conformation) in the crystal.

Following this approach, we analyzed the relationship between $K_{\mathrm{c}}$ and the polymer chain conformation in the crystalline state. From this we propose the following grouping of the polymers listed in the table:

(a) Polymers with relatively simple (i.e., without bulky side groups) molecular structure which possess a planar or weakly twisted helical conformation in the crystal (helices $1 / 1,13 / 1,14 / 1$, etc.).

(b) Polymers with a helical chain conformation (mostly vinyl polymers with helices $3 / 1$ and similar helices $7 / 2,9 / 5$, etc.). (c) Polymers with bulky side substituents crystallizing in helical conformations $11 / 3$ or $4 / 1$.

Histograms of $K_{\mathrm{c}}$ values for these three groups are shown in Figure 1. It can be seen that for (a)-group polymers the most probable values of $K_{\mathrm{c}}$ lies in the interval from 0.72 to 0.77 , while that for (b)- and (c)-groups is considerably lower. It is interesting that the histograms for (b)-series seem to be bimodal, the first peak (at $K_{\mathrm{c}}=0.68$ to 0.71 ) corresponding to polymers with mediumsize side groups (polypropylene, poly(butene-1), etc.), and the second (at $K_{\mathrm{c}}=0.62$ to 0.65 ) to polymers with long side chains (poly(4-methylhexene-1), poly(butylacrylate)s, etc.). The latter fact means that long side radicals, especially those capable of independent crystallization, hinder formation of a densely packed main-chain crystalline lattice. Finally, for (c)-group polymers the histogram is again unimodal and the peak is further shifted to lower values of $K_{\mathrm{c}}$.

From the above results one may draw two broad conclusions: that $K_{\mathrm{c}}$ depends on the polymer chain conformation in the crystal, and that $K_{\mathrm{c}}$ regularly decreases with the increase of the diameter of helical conformation (that is, chain "thickness"). Thus, it is reasonable to expect the existence of some relationship between $K_{\mathrm{c}}$
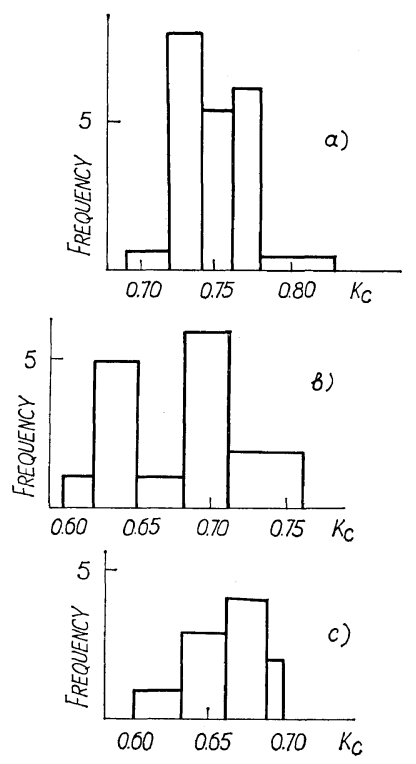

Figure 1. Histograms of $K_{c}$ values for (a)-, (b)-, and (c)-groups of polymers. 
On the Molecular Packing Density in Crystalline Polymers

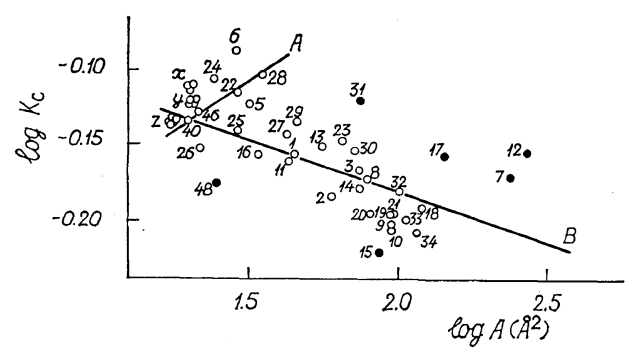

Figure 2. Dependence of $\log K_{\mathrm{c}}$ on $\log A$. Solid lines $A$ and $B$ were calculated by eq $4 a$ and $4 b$ respectively. Numbers at the data points correspond to the entries in the table. Letter " $x$ " denotes data points 38,39 , and 41 , letter " $y$ " points 35 , 36,37 , and 44 , and latter " $z$ " points $4,42,43,45$, and 49. Triangles and open circles denote EC and HC polymers respectively, while filled circles denote data points deviating more than $5 \%$ from theoretical line $\mathrm{B}$.

and the area of chain cross-section in a crystal, $A$. A double-logarithmic plot of $K_{\mathrm{c}} v s$. $A$ (in $\AA^{2}$ ) is shown in Figure 2. Values of $A$ were calculated from the tabulated unit-cell dimensions. ${ }^{12}$ It can be seen from Figure 2 that for polymers with helical chain conformation in the crystalline state (HC polymers) from groups (b) and (c) there is a definite tendency of decreasing $K_{\mathrm{c}}$ with $A$, whereas for polymers of group (a) with extended conformations of chains (EC polymers) the reverse is observed. The different behavior of $\mathrm{HC}$ and $\mathrm{EC}$ polymers is consistent with the results of our recent $\operatorname{paper}^{10}$ and can be derived analytically. For this purpose we make use of an alternative equation for $K_{\mathrm{c}}$ obtained in our recent paper, ${ }^{14}$ namely:

$$
K_{\mathrm{c}}=(\sigma / a)^{0.275}
$$

where $\sigma$ is the so-called "steric factor" ${ }^{15}$ reflecting the equilibrium stiffness of a macromolecule, and $a=A^{1 / 2}$ is the crystalline chain "thickness". ${ }^{14}$ The value of $\sigma$ can be obtained from the following empirical equations for $\mathrm{EC}$ and $\mathrm{HC}$ polymers respectively: ${ }^{10}$

$$
V_{\mathrm{c}}^{\prime}\left(\text { in } \AA^{3}\right)=\left\{\begin{array}{l}
14 \sigma \\
4.4 \sigma^{3.5}
\end{array}\right.
$$

where $V_{\mathrm{c}}{ }^{\prime}$ is the crystalline unit-cell volume per main-chain bond. Setting $V_{\mathrm{c}}{ }^{\prime}=A \cdot d$ (where $d$ is the unit-cell identity period per bond, in $\AA$ ) and substituting eq 3 into eq 2 , we obtain after some rearrangements eq 4 for EC and $\mathrm{HC}$ polymers respectively:*

$$
\log K_{\mathrm{c}}=\left\{\begin{array}{l}
-0.314+0.138 \log A \\
-0.05-0.063 \log A
\end{array}\right.
$$

The solid lines in Figure 2 were drawn according to the above equations. It is seen that for the majority of the polymers the fit of the theoretical lines to the experimental $K_{\mathrm{c}}$ values is within $5 \%$ (open circles in Figure 2), which is considered satisfactory. However, the poor fit for six polymers (filled circles in Figure 2) requires some discussion. The somewhat high values of $K_{\mathrm{c}}$ for polymeric hexa(point 7)-, octa (point 12)-, and tetra(point 17)-decenes suggest that these polymers crystallize by long side chains in addition to the main-chain crystallization. The very low value for poly(4-methylpentene-1) (point 15) suggests an unusually loosely packed crystalline structure, uncommon for the other vinyl polymers. In fact, this polymer has a crystalline density lower than that in the amorphous state at room temperature. ${ }^{12}$ We can offer no plausible explanation for the very high $K_{\mathrm{c}}$ value for poly(o-fluorostyrene) (point 31), nor for the very low $K_{\mathrm{c}}$ for poly(propylene oxide) (point 48). These might have been caused by a numerical error in the calculated crystalline densities $^{12}$ used by us in the derivation of $K_{\mathrm{c}}$ through eq 1 . We also note that the data points for the hydrogen-bonded polymers with planar chain conformation (poly(vinyl alcohol), polyamides, etc.) lie somewhat above line A for EC polymers (Figure 2). In this case, apparently the increased molecular cohesion gives an additional contribution to $K_{c}$ through the increased intermolecular packing density, as compared to polymers with the Van der Waals-type intermolecular interaction.

\section{CONCLUSIONS}

The results obtained in this work indicate that for polymers with similar types of intermolecular interaction in the crystalline state the values of the coefficient of molecular packing depend primarily on the chain conformation in the crystal. This conclusion is consistent with the

* In the derivation of eq 4, terms involving $\log d$ were neglected, since for all polymers $d \cong 1 \AA$. 


\section{P. Privalko}

data of Sakurada, et al. ${ }^{15}$ on elastic moduli of crystalline polymers, recalling that the bulk modulus (i.e., reciprocal compressibility) depends on $K_{\mathrm{c}}{ }^{2} \quad$ Moreover, our data once more confirm the concept of Volkenshtein, ${ }^{16}$ Bunn,,${ }^{17}$ and others that the polymer chain conformation in the crystalline state is determined by intramolecular interactions. Therefore, although one may speak about a very approximate (with deviations as large as $15 \%$ or so) constancy of $K_{\mathrm{c}}{ }^{4,5}$ more careful analysis suggests that this parameter is very sensitive to changes in chain conformation in the crystalline state. In fact, it can be shown that relatively small changes of $K_{\mathrm{c}}$ may lead to drastic changes in melting temperature ${ }^{18}$ kinetics, ${ }^{19}$ and energetics ${ }^{20}$ of crystalline phase formation from the melt.

Acknowledgement. I wish to express my gratitude to Professor Yu. S. Lipatov for his interest in this work.

\section{REFERENCES}

1. A. I. Kitaigorodskii, "Organic Crystallochemistry", Academy of Sciences of USSR, Moscow, 1955 (in Russian).

2. A. I. Kitaigorodskii, "Molecular Crystals," Nauka Publ., Moscow, 1971 (in Russian).

3. G. L. Slonimskii, A. A. Askadskii, and A. I. Kitaigorodskii, Vysokomol. Soedin Ser. A, 12, 494 (1970).

4. D. W. Van Krevelen (in collaboration with P. J. Hoftyzer), "Properties of Polymers," Elsevier Publ, Amsterdam, 1972.
5. M. I. Bessonov and V. E. Smirnova, Vysokomol. Soedin, Ser. B, 13, 352 (1971).

6. V. P. Lebedev, D. Ya. Tsvankin, and A. I. Kitaigorodskii, ibid., Ser. B, 13, 813 (1971).

7. B. V. Vasil"'ev, ibid., Ser. B, 15, 610 (1973).

8. V. P. Privalko, Yu. V. Pasechnik, L. I. Bezruk, and Yu. S. Lipatov, ibid., Ser. B, 15, 381 (1973).

9. V. P. Privalko in "Sintez i Fiziko-Khlm. Polimerov," N 13, Naukova Dumka Publ., Kiev, 1974, p 91.

10. V. P. Privalko, Macromolecules, 7, 111 (1973).

11. Yu. S. Lipatov and V. P. Privalko, J. Macromol. Sci.-Phys., B7, 431 (1973).

12. I. Brandrup and E. H. Immergut, Eds., "Polymer Handbook," Interscience Publ., New York, N.Y., 1966.

13. P. Corradini in "The Stereochemistry of Macromolecules" A. D. Ketley, Ed., Marcel Decker Publ., 1968, Vol. 3, p 1.

14. V.P. Privalko and Yu. S. Lipatov, Makromol. Chem. 175, 641 (1974).

15. I. Sakurada and K. Kaiji, J. Polym. Sci., Part C, 31, 57 (1970).

16. M. V. Volkenshtein, "Configurational Statistics of Polymer Chains," Academy of Sciences USSR, Moscow, 1959 (in Russian).

17. C. W. Bunn and D. R. Holmes, Discuss. Faraday Soc., 25, 95 (1958).

18. A. A. Askadskii and L. L. Slonimskii, Paper presented at XYIII All-Union Conference on Polymers, Kazan, July 1973.

19. V.P. Privalko and Yu.S. Lipatov, Kolloid.Z.Z. Polym., 251, 583 (1973).

20. V.P. Privalko, Paper presented at the IUPAC International Symposium on Macromolecules, Helsinki, 1972, Abstract III-55, p 295. 